\title{
Decoherence, Einselection and Classicality of a Macroscopic Quantum Superposition generated by Quantum Cloning
}

\author{
Francesco De Martini ${ }^{1,2}$, Fabio Sciarrino ${ }^{1}$, and Nicolò Spagnolo ${ }^{1}$ \\ ${ }^{1}$ Dipartimento di Fisica dell'Universitá "La Sapienza" and Consorzio Nazionale \\ Interuniversitario per le Scienze Fisiche della Materia, Roma, 00185 Italy \\ 2 Accademia Nazionale dei Lincei, via della Lungara 10, I-00165 Roma, Italy
}

\begin{abstract}
The high resilience to de-coherence shown by a recently discovered Macroscopic Quantum Superposition (MQS) generated by a quantum injected optical parametric amplifier (QI-OPA) and involving a number of photons in excess of $5 \times 10^{4}$ motivates the present theoretical and numerical investigation. The results are analyzed in comparison with the properties of the MQS based on $|\alpha\rangle$ and NOON states, in the perspective of the comprehensive theory of the subject by W.H.Zurek. In that perspective the concepts of "pointer state", "einselection" are applied to the new scheme.
\end{abstract}

PACS numbers:

\section{INTRODUCTION}

The short handwritten note by Einstein on the back of a greetings card sent to Max Born on the first of January 1954 may be taken as the conceptual framework of the present work: "if $\varphi_{1}$ and $\varphi_{2}$ are two solutions of the same Schrödinger equation, $\varphi=\varphi_{1}+\varphi_{2}$ is another solution of the same equation equally able to represent a possible situation. If however we are dealing with a "macrosystem" and $\varphi_{1}$ and $\varphi_{2}$ are "narrow" respect to the macrocoordinates in the vast majority of cases $\varphi$ cannot be "narrow". Narrowness respect to the macrocoordinates [i.e. macrolocalization] is a property not only independent of the principles of quantum mechanics but also incompatible with them" [1].

As we can see since the early decades of Quantum Mechanics the counter-intuitive properties associated with the superposition state of macroscopic objects and the problem concerning the "classicality" of quantum macrostates were the object of an intense debate epitomized in 1935 by the celebrated "Schrödinger Cat paradox" 2, 3]. In particular, the actual feasibility of such quantum object has always been tied to the alleged infinitely short persistence of its quantum coherence, i.e. of its overwhelmingly rapid "decoherence". In modern times the latter property, establishing a rapid merging of the quantum rules of microscopic systems into classical dynamics, has been interpreted as a consequence of the entanglement between the macroscopic quantum system with the environment [4, 5]. By tracing over the environmental variables in the final calculations, generally the pure quantum state decays irreversibly towards a probabilistic classical mixture [6]. Recently, the general interest for decoherence has received a renewed interest in the framework of quantum information theory where it plays a fundamental detrimental role since it conflicts with the experimental realization of the quantum computer or of any quantum device bearing any relevant complexity [7]. In this respect a large experimental effort has been devoted recently to the implementation of Macroscopic (i.e. many-particle) Quantum Superpositions states (MQS), adopting photons, atoms and electrons in superconducting devices. Particular attention has been devoted to the realization of the MQS involving "coherent states" of light, which exhibits interesting and elegant Wigner function representations [8]. The most notable results of this experimental effort have been reached with atoms interacting with microwave fields trapped inside a cavity [9, 10] or for freely propagating fields [11]. However, in spite of the long lasting efforts spent in these endeavors, in these realizations the MQS has always proved to be so fragile that even the loss of a single particle was found to be able to spoil any possibility of a direct observation of its quantum properties. Precisely on the basis of these negative results in many scientific communities (and also within some influential editorial teams) grew the opinion that the "Schrödinger Cat" is indeed an ill defined and then avoidable concept since it fundamentally lacks of any directly observable property [6].

In spite of these conclusions, very recently a new kind of MQS involving a number of particles $N$ in excess of $5 \times 10^{4}$ has been realized allowing the direct observation of entanglement between a microscopic (Micro-) and a macroscopic (Macro-) photonic state and showing a very high resilience to decoherence by coupling with environment [12]. Precisely, the MQS was generated by a quantum-injected optical parametric amplifier (QI-OPA) seeded by a single-photon belonging to an EPR entangled pair. We emphasize here that the reported QI-OPA can be considered for the present purpose as a paradigmatic system consisting of the simplest realizable "optimal phase-covariant quantum cloning machine" [13, 14]. Indeed, precisely the process of "quantum cloning" was there responsible for the transfer of the entanglement and the superposition properties of a pure single-particle qubit into a multiparticle MQS. In other words, the QIOPA encoded "optimally" into a Macro-state the information associated with the input Microstate, a seed qubit [15, 16, 17, 18, 19]. By this device, that includes an Orthogonality Filter (O-Filter) for enhanced state discrimination, the Micro-Macro state non-separability was successfully tested and the Micro-Macro violation of the Bell'inequalities for Spin-1 excitations was attained 


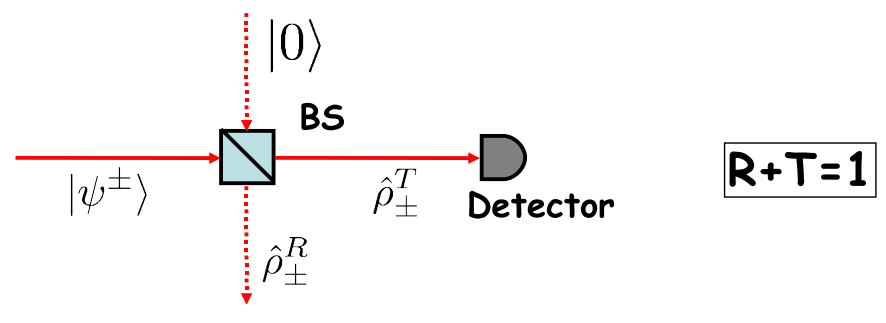

FIG. 1: (Color online) Schematization of the decoherence model by a linear beam-splitter of transmittivity $\mathrm{T}$.

[12, 20]. In view of this peculiar, striking behavior, we felt that a careful analysis of the decoherence of this novel MQS device was necessary. The present approach to decoherence will be cast within the useful framework developed in the past by Wojciech H.Zurek[21]. Accordingly, the systems we shall consider will be characterized according to the concepts of "information flow", "pointer states", "einselection". Precisely, according to Zurek's definition, the "pointer states" of any detector, or of any open quantum system, consist of a preferred basis that is selected, i.e. "einselected" by the characteristic detector - environment interaction. When expressed in terms of these states, the correct density matrix for the detector - system combination can be obtained by standard Schrödinger equation theory without having to appeal to the von Neumann's nonunitary "reduction" process. These privileged pointer states represent the natural behavior of the detector's "pointer" expressing the physical outcome of any measurement process. The present work is intended to provide a new insight in the elusive, fundamental problem of decoherence and, at last, it will provide a sensible reply to the argument expressed in the Einstein letter to Born. Hopefully, it may also lead to a revision of several prejudices about MQS.

Let's summarize the content of the present paper. In Section II, we introduce the criteria adopted to characterize the resilience to decoherence of any MQS while, in order to test the validity of our approach, these criteria are applied in Section III to the case of the "coherent" Glauber's - state MQS. We obtain an universal function which is in agreement to the expected exponential decrease of coherence, induced by losses, peculiar of this class of states. The central point of the paper is addressed in Section IV where a thourough computer analysis of the decoherence affecting the MQS generated by the process of optimal phase-covariant quantum cloning of a single-photon state. The applicaton of the criteria here introduced show the very high resilience to losses of this MQS. Finally, by the conclusive Section V the scope of the work is considered on a broader perspective involving some relevant, basic quantum mechanical issues.

\section{DEFINITIONS AND CRITERIA}

In this Section we introduce the method we intend to adopt in order to provide a consistent investigation of the "resilience to decoherence" of any MQS. Precisely, we introduce first the criteria, based on the concept of "distinguishability" between two orthogonal quantum states and the related "degree of coherence" of a MQS involving the same states. The parameter here introduced, the "Bures distance" is assumed as the "merit figure" expressing the persistance of quantum effects when decoherence process progressively randomizes the relative phase between the two components of the MQS. Finally, we describe the model adopted to simulate the transmission of the analyzed field over a "lossy channel".

Criteria for macroscopic superposition. In order to distinguish between two different quantum states, let us introduce the definition of "distance" $D$ in the Hilbert space, i.e. a parameter which expresses quantitatively the overlap of two generic states $\widehat{\rho}$ and $\widehat{\sigma}$ via the "fidelity" $\mathcal{F}(\widehat{\rho}, \widehat{\sigma})=\operatorname{Tr}\left(\sqrt{\widehat{\rho}^{\frac{1}{2}} \widehat{\sigma} \widehat{\rho}^{\frac{1}{2}}}\right)$ being $0 \leq \mathcal{F} \leq 1$, where $\mathcal{F}=1$ for $\widehat{\rho}=\widehat{\sigma}$, and $\mathcal{F}=0$ for orthogonal states [22]. This quantity is adopted to define the "Bures distance", a metric in the state space: $D(\widehat{\rho}, \widehat{\sigma})=\sqrt{1-\mathcal{F}(\widehat{\rho}, \widehat{\sigma})}$ [23, 24].

Distinguishability, MQS "Visibility". Let's characterize two macroscopic states $\left|\phi_{1}\right\rangle$ and $\left|\phi_{2}\right\rangle$ and the corresponding MQS's: $\left|\phi^{ \pm}\right\rangle=\frac{\mathcal{N}_{ \pm}}{\sqrt{2}}\left(\left|\phi_{1}\right\rangle \pm\left|\phi_{2}\right\rangle\right)$ by adopting two criteria. I) The "Distinguishability" between $\left|\phi_{1}\right\rangle$ and $\left|\phi_{2}\right\rangle$ expressed by: $D\left(\left|\phi_{1}\right\rangle,\left|\phi_{2}\right\rangle\right)$. II) The "Visibil$i t y "$, i.e. "degree of orthogonality" of two MQS's $\left|\phi^{ \pm}\right\rangle$expressed by $D\left(\left|\phi^{+}\right\rangle,\left|\phi^{-}\right\rangle\right)$. Indeed, the value of the MQS visibility depends exclusively on the relative phase of the component states: $\left|\phi_{1}\right\rangle$ and $\left|\phi_{2}\right\rangle$. The parameter $D$ expresses the ability of an observer to discriminate between two initially orthogonal states, $D\left(\left|\phi^{+}\right\rangle,\left|\phi^{-}\right\rangle\right)=1$, after propagation in a "lossy channel" where the relative phase of $\left|\phi_{1}\right\rangle$ and $\left|\phi_{2}\right\rangle$ progressively randomizes leading to a fully mixed state: $D\left(\left|\phi^{+}\right\rangle,\left|\phi^{-}\right\rangle\right)=0$.

As we shall see later in the paper, the physical interpretation of $D\left(\left|\phi^{+}\right\rangle,\left|\phi^{-}\right\rangle\right)$as "Visibility" of a superposition $\left|\phi^{ \pm}\right\rangle$is legitimate insofar as the component states of the corresponding superposition, $\left|\phi_{1}\right\rangle$ and $\left|\phi_{2}\right\rangle$ may be defined, at least approximately, as "pointer states" or "einselected states" [21]. Within the set of the eigenstates characterizing any quantum system the pointer states are defined as the ones least affected by the external noise and that are highly resilient to decoherence. In other words, the pointer states are "quasi classical" states which realize the minimum flow of information from (or to) the System to (or from) the Environment. Amongst the reasonable criteria of classicality, such as the ones based on "purity" of the macrostates or on their "predictability", discussed by W. H. Zurek in Reviews of Modern Physics [21], the distinguishability criterion adopted in the present work is likely to be related to the "distinguishability sieve" suggested by B.W.Schumaker 
in 1999 and referred to in that paper as a "private communication".

The lossy Channel is modelled in the present analysis by a generic linear beam-splitter (BS) with transmittivity $T$ and reflectivity $R=1-T$ acting on a generic quantum state associated with a single mode beam: Fig 1 [25, 26]. As usually done with photons, the scattering provided by BS (BS-scattering) is assumed to represents well the decoherence process, the one that provides the flow of information from the system to the environment. As it is well known, the BS-scattering is also generally assumed to model the necessarily limited quantum efficiency of any realistic photodetector, $Q E<1[25]$. Then our present interpretation may be thought of as to include conceptually the latter effect into an overall decoherence scheme involving only ideal detectors $(Q E=1)$ at the end of the measurement chain. The calculation of the output density matrix consists of the $T$-dependent BS-scattering transformation on the input state, and of the evaluation of the partial trace (R-trace) of the emerging field on the reflected mode, i.e. the loss variables. The aim of the paper is to study the evolution of two Macro-states $\left|\phi_{1}\right\rangle$ and $\left|\phi_{2}\right\rangle$ and of their superpositions $\left|\phi_{ \pm}\right\rangle$by the size of the corresponding $D\left(\left|\phi_{1}\right\rangle,\left|\phi_{2}\right\rangle\right)$, $D\left(\left|\phi^{+}\right\rangle,\left|\phi^{-}\right\rangle\right)$as a function of the parameters $R$ (or $T$ ) of the lossy channel. We start by analyzing the well known MQS generated by the Glauber's coherent states $[9,10]$. Then we shall consider somewhat extensively the QI-OPA solution, which is at the focus of the present analysis. At last, the comparison between the two cases will provide an insightful assessment of our results.

\section{QUANTUM SUPERPOSITION OF GLAUBER'S COHERENT STATES}

The method introduced in the previous Section for studying the resilience to decoherence of macroscopic states and their quantum superposition is here applied to the MQS of "coherent" Glauber's - states. The properties of the states have been widely studied in the past [9, 10, 27] and represent a crucial test to verify the validity of our present approach. The MQS made by coherent states $\left|\phi_{1,2}\right\rangle=| \pm \alpha\rangle$ is defined here as: $\left|\phi^{ \pm}\right\rangle=\frac{\mathcal{N}}{\sqrt{2}}(|\alpha\rangle \pm|-\alpha\rangle)$, where $\mathcal{N}$ is a normalization quantity [27]. In the specific case of input coherent states $| \pm \alpha\rangle$, the application of the above loss model leads after BS - scattering to the output coherent-state density matrix: $\widehat{\rho}_{ \pm \alpha}^{T}=| \pm \sqrt{T} \alpha\rangle\langle \pm \sqrt{T} \alpha|$, i.e. the "decoherence" due to scattering doesn't change the structure of the states. The distance between the two states with opposite phase is easily found [25] : $D(|\sqrt{T} \alpha\rangle,|-\sqrt{T} \alpha\rangle)=$ $\sqrt{1-e^{-2 T|\alpha|^{2}}}$, a value close to 1 for an average number of transmitted particles $T|\alpha|^{2}$ where: $0<T|\alpha|^{2}<|\alpha|^{2}$ Fig2 2(d). In this regime the coherent states $| \pm \alpha\rangle$ keep their mutual distinguishability through the lossy channel and comply with Zurek's definition of "pointer states".
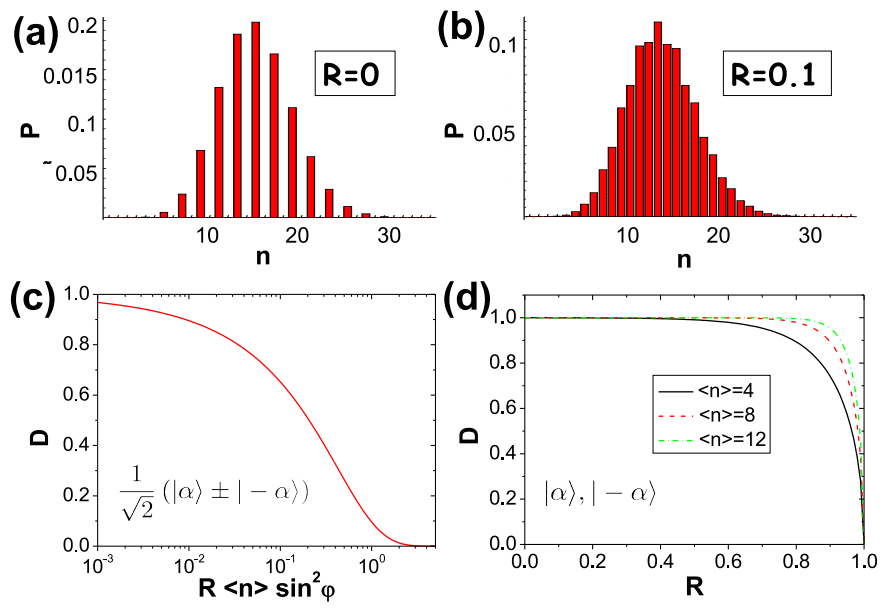

FIG. 2: (Color online) (a)-(d): Plot of the distribution of the number of photons in the $\left|\phi^{+}\right\rangle$state for $\alpha=4$, corresponding to an average number of photons $\langle n\rangle=16$, for reflectivities $R=0$ (fig.2-a) and $R=0.1$ (fig.2-b). (c): Plot of the universal curve that describes the distance between $\left|\phi^{+}\right\rangle$and $\left|\phi^{-}\right\rangle$ after losses as a function of $R\langle n\rangle \sin ^{2} \varphi$. This universal curve is valid for the more general case of the $\left|\phi_{\varphi}^{ \pm}\right\rangle$states when plotted as a function of $R\langle n\rangle \sin ^{2} \varphi$. (d): Distance between the coherent $|\alpha\rangle$ and $|-\alpha\rangle$ states as a function of the reflectivity $R$ for different values of $\langle n\rangle$. From lower to the upper curves, the lines correspond to increasing values of $\langle n\rangle$ as indicated in the legend.

Let's now consider the MQS "Visibillity". The R-reduced density matrices after the BSscattering have the general form: $\hat{\rho}_{T \pm}=$ $\frac{1}{2}\left(|\beta\rangle\langle\beta|+|-\beta\rangle\langle-\beta| \pm e^{-2 R|\alpha|^{2}}(|-\beta\rangle\langle\beta|+| \beta\rangle\langle-\beta|)\right)$ with $|\beta\rangle=|\alpha \sqrt{T}\rangle$. For the coherent state MQS with no losses $(T=1)$, the distribution in the Fock space exhibits only elements with an even number of photons for $\left|\phi^{+}\right\rangle$ or an odd number of photons for $\left|\phi^{-}\right\rangle$. The quantum superposition of these states is exclusively attributable to this very peculiar "comb" structure of the Fock spectrum. This structure is indeed extremely fragile under the effect of losses since the R-trace operation must be carried out in the space of the non-orthogonal coherent-states. This is shown in Fig/2(a)-(b) for increasing values of the particle loss. The MQS Visibility of these states can be evaluated analytically in closed form and is found extremely sensitive to decoherence [28]:

$$
D=\sqrt{1-\sqrt{1-e^{-4 R|\alpha|^{2}}}}
$$

i.e., $D(x) \simeq e^{-2 x}$ being: $x \equiv R<n>=R|\alpha|^{2} \geq 1$, the average number of lost photons. The loss of 1 photon, on the average, leads to the MQS Visibility value: $D=0.096$, and then to the practical cancellation of any detectable interference effects involving $\hat{\rho}_{\phi^{ \pm}}^{T}$. This is fully consistent with the experimental observations 
[9, 10]. The previous calculations generalize to the general coherent state MQS: $\left|\phi_{\varphi}^{ \pm}\right\rangle=\frac{\mathcal{N}_{\varphi}^{ \pm}}{\sqrt{2}}\left(\left|\alpha e^{\imath \varphi}\right\rangle \pm\left|\alpha e^{-\imath \varphi}\right\rangle\right)$ by substituting $|\alpha|^{2}$ with $|\alpha|^{2} \sin ^{2} \varphi$. Hence, we can summarize the theoretical results for the MQS Visibility by tracing the unique function: $D\left(\left|\phi_{\varphi+}\right\rangle,\left|\phi_{\varphi-}\right\rangle\right)=D(x)$ with $x=R|\alpha|^{2} \sin ^{2} \varphi$, shown in Fig. 2-(c). We consider this "universal" function an additional important property of the "coherent states" (not previously discovered, to our knowledge). Note that the function $D(x)$ approaches its minimum value with zero slope: $S l=\lim _{R \rightarrow 1}|d D(x) / d x|=0$.

As noted by Gunnar Bjork [29], a similar behavior is obtained by applying the present decoherence model to the entangled two-mode number states, i.e. the states $\left|\phi_{N 1}\right\rangle=|N 0\rangle$ and $\left|\phi_{N 2}\right\rangle=|0 N\rangle$ and to their superpositions, called NOON states: $\left|\phi_{N \pm}\right\rangle=\frac{\mathcal{N}^{ \pm}}{\sqrt{2}}(|N 0\rangle \pm$
$|0 N\rangle$ ), for large $\mathrm{N}$ [30]. After R-tracing, the distance corresponding to the Fock-states $|N 0\rangle$ and $|0 N\rangle$ above is found to scale as $\mathcal{D}\left(\phi_{N 1}, \phi_{N 2}\right)=\sqrt{1-R^{N}}$, while the distance corresponding to their superpositions is found: $\mathcal{D}\left(\phi_{N \pm}\right)=\sqrt{(1-R)^{N}}$. Then it turns out that, while $\mathcal{D}\left(\phi_{N 1}, \phi_{N 2}\right) \simeq 1$ for $\langle n\rangle \ll N$, the total number of particles, $\mathcal{D}\left(\phi_{N_{ \pm}}\right)$drops to zero as soon as $R\langle n\rangle \simeq 1$, with the cancellation of the visibility after loss of a single photon on the average. This behaviour allows to identify $\phi_{N 1}, \phi_{N 2}$ with the "pointer states" of the system, which show a slow decoherence rate and can in principle be discriminated bu a suitable measurement even in a high lossy transmission channel similarly to the $|\alpha\rangle$ states. On the contrary, the quantum superpositions $\phi_{N \pm}$ are fragile under decoherence as the $(|\alpha\rangle \pm|-\alpha\rangle)$ states are.

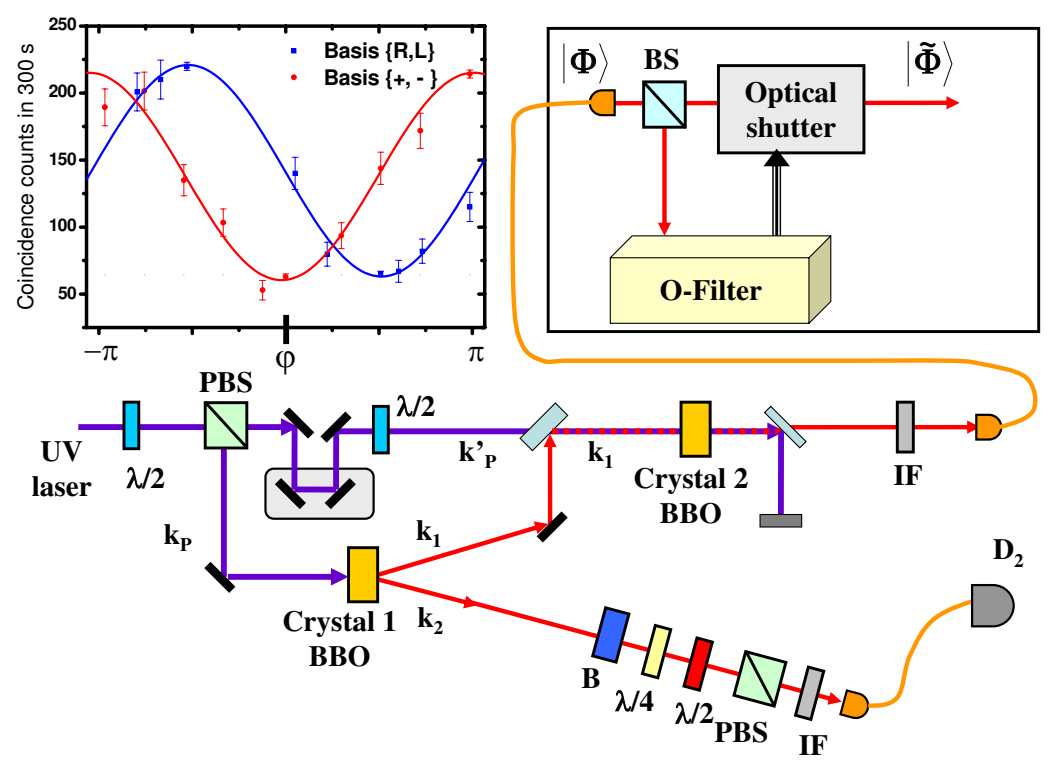

FIG. 3: (Color online) Scheme of the experimental setup for the observation of entanglement between a microscopic and a macroscopic system. The main UV laser beam provides the OPA excitation field beam at $\lambda=397.5 \mathrm{~nm}$. A type II BBO crystal (crystal 1: C1) generates pair of photons with $\lambda=795 \mathrm{~nm}$. In virtue of the EPR non-local correlations established between the modes $\mathbf{k}_{1}$ and $\mathbf{k}_{2}$, the preparation of a single-photon on mode $\mathbf{k}_{1}$ with polarization state $\vec{\pi}_{\varphi}$ is conditionally determined by detecting a single-photon after proper polarization analysis on the mode $\mathbf{k}_{2}$ (polarizing beamsplitter (PBS), $\lambda / 2$ and $\lambda / 4$ waveplates, Soleil-Babinet compensator (B), interferential filter (IF)). The photon belonging to $\mathbf{k}_{1}$, together with the pump laser beam $\mathbf{k}_{p}^{\prime}$, is fed into an high gain optical parametric amplifier consisting of a NL crystal 2 (C2), cut for collinear type-II phase matching. The fields are coupled to single-mode fibers. For more details refer to [12]. Left inset: Experimental results of the interference fringe pattern between the microscopic $\mathbf{k}_{2}$ and the macroscopic $\mathbf{k}_{1}$ fields [12]. Square data corresponds to the fringe pattern in the $\{R, L\}$ basis, circular data in the $\{+,-\}$ basis. Right Inset: O-Filtering process obtained by an "idle" measurement apparatus. A portion of the wave-function $(\simeq 10 \%)$ is measured and analyzed by an O-Filter driving a fast e-optical shutter.

In conclusion, note that the very high resilience to

decoherence shown by the "pointer states" just consid- 
ered parallels a very high sensitivity to decoherence of the quantum superpositions of the same states. As we shall see, this important property is at variance with the behavior of the "pointer states" realized by Quantum Cloning.

\section{QUANTUM SUPERPOSITION BY OPTIMAL PHASE-COVARIANT QUANTUM CLONING.}

In this Section we address the central point of the paper. As said, recent experimental results [12] showed that quantum properties, such as quantum entanglement, can be still observed in macroscopic system of $\approx 10^{4}$ particles even after the transmission over a lossy channel and the detection by a measurement apparatus with non-unitary detection efficiency. In facts, according to the experimental evidence and in agreement with the theory reported in this Section, an exceedingly high resilience to decoherence is common both to the "pointer" macrostates gen- erated by the QI-OPA system and, by the same amount, to all quantum superpositions of the same states [12]. In order to give a theoretical insight on this task, we apply the method of Sec.II to the amplified single photon qubits by a collinear QI-OPA, i.e. the simplest "optimal phasecovariant quantum cloning machine". We first derive the expression of the density matrix of these multi-photon states after propagation over a lossy channel, for several input polarization states of the injected qubit. The evolution of the photon-number distributions leads to a first insightful picture of the effects of decoherence and introduces the more quantitative results given by the $\mathrm{Bu}$ res distance. The latter is then evaluated by performing a numerical calculation of the quantum fidelity between the exact density matrices. Furthermore, the same procedure is applied to the recently discovered O-Filter device 12] showing that this device can substantially reduce the decoherence effects on the MQS visibility, at the cost of discarding a part of the data.
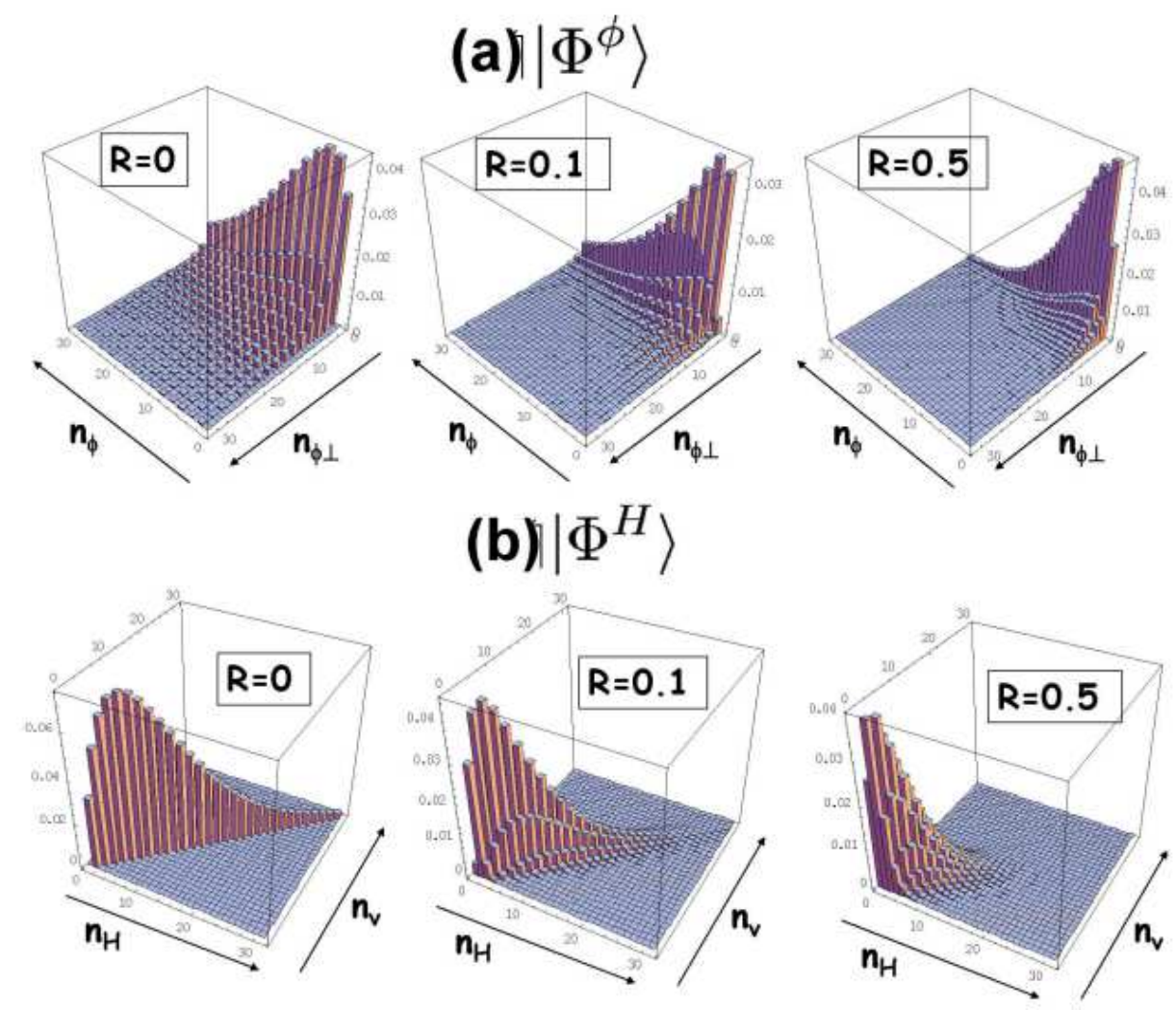

FIG. 4: (Color online) (a) Probability distribution in the Fock space $\left(n_{\phi}, n_{\phi_{\perp}}\right)$ for the amplified $\left|\Phi^{\phi}\right\rangle$ state of a generic equatorial qubit for different values of the transmittivity. (b) Probability distribution in the Fock space $\left(n_{H}, n_{V}\right)$ for the amplified $\left|\Phi^{H}\right\rangle$ state for different values of the transmittivity. All distributions refer to a gain value of $g=1.5$, corresponding to an average number of photons $\langle n\rangle \approx 19$.

Let us begin by describing the system under investigation. The QI-OPA device is represented in Fig 3 to- gether with the interference fringes of the Macrostate 
quantum superpositions obtained in a recent experiment (Left Inset) [12, 15]. Remind that the phase-covariant process clones identically and "optimally", i.e. with the minimal "squeezed - vacuum noise" allowed by the nocloning theorem and implied by parametric amplification, all input qubits belonging on the Poincaré sphere to the "equatorial" plane orthogonal to the polarization basis $\left\{\vec{\pi}_{H}, \vec{\pi}_{V}\right\}$. Here $\mathrm{H}$ and $\mathrm{V}$ refer to horizontal and vertical optical polarizations [13, 14]. The interaction Hamiltonian is: $\widehat{\mathcal{H}}_{\text {coll }}=\imath \hbar \chi \widehat{a}_{H}^{\dagger} \widehat{a}_{V}^{\dagger}+$ h.c. when expressed in the basis $\left\{\vec{\pi}_{H}, \vec{\pi}_{V}\right\}$, or $\widehat{\mathcal{H}}_{\text {coll }}=\frac{\imath \hbar \chi}{2} e^{-\imath \phi}\left(\widehat{a}_{\phi}^{\dagger 2}-e^{\imath 2 \phi} \widehat{a}_{\phi_{\perp}}^{\dagger 2}\right)$ when expressed in any equatiorial basis $\left\{\vec{\pi}_{\phi}, \vec{\pi}_{\phi \perp}\right\}$, where: $\vec{\pi}_{\phi}=2^{-\frac{1}{2}}\left(\vec{\pi}_{H}+e^{\imath \phi} \vec{\pi}_{V}\right)$. Two relevant "equatorial" bases $\left\{\vec{\pi}_{+}, \vec{\pi}_{-}\right\}$and $\left\{\vec{\pi}_{R}, \vec{\pi}_{L}\right\}$ correspond respectively to the phase sets $\phi=\{0, \pi\}$ and $\phi=\{\pi / 2,3 \pi / 2\}$. By direct calculation, obtained applying the unitary cloning operator $\hat{U}=e^{-\frac{2 \mathcal{H}_{i n t} t}{\hbar}}$, the output states for an injected qubit $\pi=\{H, V\}$ is found to have the Fock-state expansion [12]:

$$
\left|\Phi^{\pi}\right\rangle=\hat{U}|\pi\rangle=\frac{1}{C^{2}} \sum_{i=0}^{\infty} \Gamma^{i} \sqrt{i+1}\left|(i+1) \pi, i \pi_{\perp}\right\rangle
$$

where the ket $\left|n \pi, m \pi_{\perp}\right\rangle$ represents the number state with $n$ photons with $\pi$ polarization and $m$ photons with $\pi_{\perp}$ polarization. With the same procedure, for an injected equatorial qubit the optimally amplified state is:

$$
\left|\Phi^{\phi}\right\rangle=\hat{U}|\phi\rangle=\sum_{i, j=0}^{\infty} \gamma_{i j}\left|(2 i+1) \phi,(2 j) \phi_{\perp}\right\rangle
$$

where $\gamma_{i j}=\frac{1}{C^{2}}\left(e^{-\imath \varphi} \frac{\Gamma}{2}\right)^{i}\left(-e^{\imath \varphi} \frac{\Gamma}{2}\right)^{j} \frac{\sqrt{(2 i+1) !} \sqrt{(2 j) !}}{i ! j !}$. In these expressions $C=\cosh g$ and $\Gamma=\tanh g$, where $\mathrm{g}$ is the non linear gain of the amplifier. Consider the "equatorial" macrostates $\left|\Phi^{+}\right\rangle$and $\left|\Phi^{-}\right\rangle$corresponding respectively to $\phi=0$ and $\phi=\pi$, and let's assume them provisionally as "pointer macrostates" [21].
The general expression of any macroqubit lying on the equatorial plane may be taken as a MQS of $\left|\Phi^{+}\right\rangle$and $\left|\Phi^{-}\right\rangle:\left|\Phi^{\phi}\right\rangle=e^{-i \phi / 2}\left[\cos (\phi / 2)\left|\Phi^{+}\right\rangle+i \sin (\phi / 2)\left|\Phi^{-}\right\rangle\right]$. Assume now, for the sake of definiteness, the two independent MQS's identified by the new "equatorial" basis $\left\{\left|\Phi^{R}\right\rangle,\left|\Phi^{L}\right\rangle\right\}:\left|\Phi^{R}\right\rangle=\frac{\mathcal{N}_{t}}{\sqrt{2}}\left(\left|\Phi^{+}\right\rangle+i\left|\Phi^{-}\right\rangle\right)$and $\left|\Phi^{L}\right\rangle=$ $\frac{\mathcal{N}_{ \pm}}{\sqrt{2}}\left(\left|\Phi^{+}\right\rangle-i\left|\Phi^{-}\right\rangle\right)$[17]. Indeed, owing to linearity and to the phase-covariance of the cloning process, each basis set of macrostates lying on the equatorial plane is a quantum superposition of any other macrostate set lying on that same plane. Therefore the distinguishability of $\left\{\left|\Phi^{+}\right\rangle,\left|\Phi^{-}\right\rangle\right\}$expressed by the distance $D\left(\left|\Phi^{+}\right\rangle,\left|\Phi^{-}\right\rangle\right)$ coincides with the MQS Visibility of any superpositions $\left|\Phi^{\phi}\right\rangle$, as for instance of $\left|\Phi^{R}\right\rangle$ or $\left|\Phi^{L}\right\rangle$ :

$$
D\left(\left|\Phi^{R}\right\rangle,\left|\Phi^{L}\right\rangle\right)=D\left(\left|\Phi^{+}\right\rangle,\left|\Phi^{-}\right\rangle\right)
$$

In conclusion, the peculiar phase-covariant symmetry of the cloning process allows to identify the equatorial plane of the Poincarè sphere of the macroqubits as a preferred Hilbert subspace in which the assumed "pointer" macrostates as well as any MQS contributed by them are affected by the same decoherence process and are then granted by an identical einselection property [21]. As already noted, all these important properties of the QI-OPA system are at variance with the general case considered by Einstein in the quoted letter to Born and with the particular case with coherent or NOON states just considered.

In order to assess the einselected status of the macrostates at hand and the related MQS's, let's now consider the decoherence induced by BS-scattering and the corresponding Fock space spectra. The first step is the calculation of the explicit form of the density matrix after transmission over the lossy channel for the equatorial Macro-qubits. We report here their explicit form:

$$
\begin{aligned}
& \left(\hat{\rho}_{T}^{\phi}\right)_{i j k q}=\sum_{m, n=0}^{\infty} \frac{1}{C^{4}}\left(\frac{\Gamma}{2}\right)^{\frac{i+j+k+q}{2}+m+n-1}(-1)^{\frac{j+q}{2}+n}\left(e^{\imath \varphi}\right)^{\frac{j+k-i-q}{2}} \frac{\sqrt{(i+m) !(j+n) !(k+m) !(q+n) !}}{\left(\frac{i+m-1}{2}\right) !\left(\frac{j+n}{2}\right) !\left(\frac{k+m-1}{2}\right) !\left(\frac{q+n}{2}\right) !} \\
& (\sqrt{T})^{i+j+k+q}(R)^{m+n}\left[\left(\begin{array}{c}
i+m \\
m
\end{array}\right)\left(\begin{array}{c}
j+n \\
n
\end{array}\right)\left(\begin{array}{c}
k+m \\
m
\end{array}\right)\left(\begin{array}{c}
q+n \\
n
\end{array}\right)\right]^{\frac{1}{2}} f(i, k, m) h(j, q, n)
\end{aligned}
$$

corresponding to the $\left|i \phi, j \phi_{\perp}\right\rangle\left\langle k \phi, j \phi_{\perp}\right|$ matrix element, where $f(i, k, m)$ and $h(j, q, n)$ express the constraints over the parity of the indexes $\{i, j, k, q\}$ according to:

$$
f(i, k, m)= \begin{cases}1 & \text { if } i \bmod (2)=k \bmod (2) \neq m \bmod (2) \\ 0 & \text { otherwise }\end{cases}
$$




$$
h(j, q, n)= \begin{cases}1 & \text { if } j \bmod (2)=q \operatorname{Mod}(2)=n \bmod (2) \\ 0 & \text { otherwise }\end{cases}
$$

We stress that both these constraints and the sums $\sum_{m} \sum_{n}$ derive from the interaction of the initial wave function with the beam-splitter $\hat{U}_{B S}$ [25] and the subsequent R-tracing, i.e. the partial trace over the reflected mode of $U_{B S}\left|\Phi^{\phi}\right\rangle$. These sums however can be further rearranged by use of the Hyper-geometric functions ${ }_{2} F_{1}(\alpha, \beta ; \gamma ; z) 31$.

With an analogous procedure, the $\{\pi=H, V\}$ states after the R-tracing are described by the following density matrix:

$$
\begin{aligned}
& \hat{\rho}_{T}^{H}=\sum_{i=1}^{\infty} \sum_{j=0}^{i-1} \sum_{k=0}^{\infty}\left(\sum_{p=0}^{\infty} \bar{\gamma}_{i j k ; p}\right)\left|i \pi, j \pi_{\perp}\right\rangle\left\langle k \pi,(k+j-i) \pi_{\perp}\right|+ \\
& +\sum_{i=0}^{\infty} \sum_{j=i}^{\infty} \sum_{k=0}^{\infty}\left(\sum_{p=j+1-i}^{\infty} \bar{\gamma}_{i j k ; p}\right)\left|i \pi, j \pi_{\perp}\right\rangle\left\langle k \pi,(k+j-i) \pi_{\perp}\right|
\end{aligned}
$$

where the coefficients $\bar{\gamma}_{i j k ; p}$ are:

$$
\begin{gathered}
\bar{\gamma}_{i j k ; p}=\frac{\Gamma^{2 p+i+k-2}}{C^{4}} \sqrt{p+i} \sqrt{p+k} T^{k+j} R^{2 p+i-1-j} \\
{\left[\left(\begin{array}{c}
p+i \\
i
\end{array}\right)\left(\begin{array}{c}
p+i-1 \\
j
\end{array}\right)\left(\begin{array}{c}
p+k \\
k
\end{array}\right)\left(\begin{array}{c}
p+k-1 \\
k+j-1
\end{array}\right)\right]^{\frac{1}{2}}}
\end{gathered}
$$

Fig 4 reports the distribution in the Fock space $P\left(n_{\phi}, n_{\phi \perp}\right)$ corresponding to different macrostates $\left|\Phi^{\phi}\right\rangle$ for different values of the reflectivity $R$. For the unperturbed states $(\mathrm{R}=0)$, as shown by Fig 4 (a), each "equatorial" macrostate $\left|\Phi^{\phi}\right\rangle$, evaluated by Eq.(3) exhibits a typical comb structure, i.e. the spectrum of Fock space contains only nonvanishing terms with a specific parity, in particular odd photon numbers for $\vec{\pi}_{\phi}$ polarization and even photon numbers for its orthogonal $\vec{\pi}_{\phi \perp}$. On the other hand, the amplified $\left\{\left|\Phi^{H, V}\right\rangle\right\}$ states, that are not equatorial states, are characterized by a diagonal distribution. When losses are inserted (Fig,4), these peculiar properties are progressively cancelled, as for the Glauber's - states MQS considered in Section III. However, the distributions corresponding to initially orthogonal macroqubits remain distinguishable even after losses only for the equatorial $\left|\Phi^{\phi}\right\rangle$ states (Fig 4 (a)), since most of the events are localized in different Fock-space zones, i.e. where the number of amplified photons bearing the same polarization $\vec{\pi}$ of the injected qubit is substantially higher than the ones which are in the orthogonal $\vec{\pi}_{\pi}$. This indeed corresponds to the optimal quantum cloning feature of the QIOPA, that in the high gain regime survives in lossy schemes. This latter feature is absent in the $\left|\Phi^{H, V}\right\rangle$ macroqubits since the $\left\{\vec{\pi}_{H}, \vec{\pi}_{V}\right\}$ basis, is not "equatorial" and then it doesn't correspond to an "optimal" cloning by a collinear QI-OPA.

The visibility $D(x)$ of the MQS belonging to the "equatorial" subspace $\left\{\left|\Phi^{\phi}\right\rangle,\left|\Phi^{\phi \perp}\right\rangle\right\}$ has been evaluated numerically as function of the average lost photons: $x \equiv$ (a)

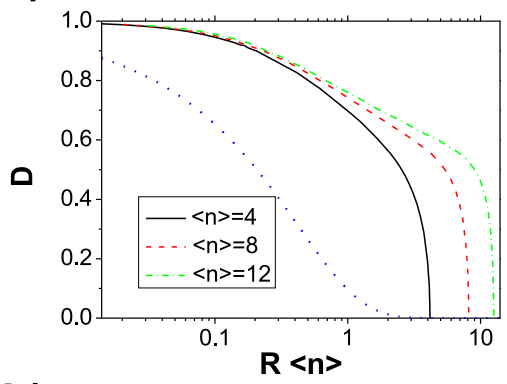

(b)

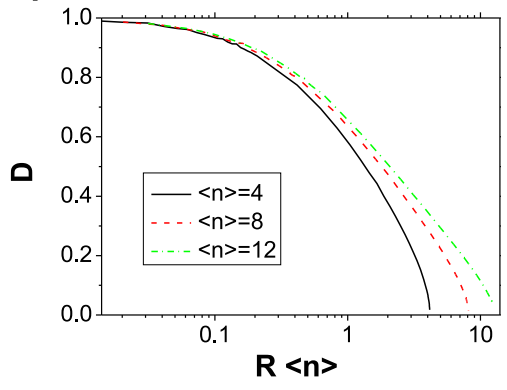

FIG. 5: (Color online) (a) Numerical evaluation of the distance $D(x)$ between two orthogonal equatorial macro-qubits $\left|\Phi^{\phi, \phi_{\perp}}\right\rangle$ as function of the average lost particle $\left.x=R<n\right\rangle$, plotted in a logarirhmic scale. Black straight line refers to $g=0.8$ and hence to $\langle n\rangle \approx 4$, red dashed middle line to $g=1.1$ and $\langle n\rangle \approx 8$, green dash-dotted upper line to $g=1.3$ and $\langle n\rangle \approx 12$. The blue dotted lower line corresponds to the function $D(x)$ for coherent state MQS, universal for any value of $\langle n\rangle$. (b) Numerical evaluation of the Bures distance between $\left|\Phi^{H}\right\rangle$ and $\left|\Phi^{V}\right\rangle$ for the same values of the gain of (a). Black straight lower line refers to $g=0.8$ and hence to $\langle n\rangle \approx 4$, red dashed middle line to $g=1.1$ and $\langle n\rangle \approx 8$, green dash-dotted upper line to $g=1.3$ and $\langle n\rangle \approx 12$. We note the faster decrease of the distance with respect to the equatorial case.

$R<n>$. More specifically, we calculated the quantum fidelity between these state by a numerical program which re-organized the density matrix of eqq.(5) in a linear algebraic matrix form. The fidelity, and hence the Bures distance, was then estimated by using algebraic numerical routines. The results for different values of the gain are reported in Fig.5.(a). Note that for small values of $x$ the decay of $D(x)$ is far slower than for the coherent state case shown in Fig 2-(e) and reproduced again in Fig 5 for comparison (dotted line). A direct comparison with the previous case shows that the resilience of QIOPA amplified states increases with a higher number $N$, the total output number of particles in the primary beam. Furthermore, after a common inflexion point at $D \sim 0.5$ the function $D(x)$ drops to zero for $R=1$ and then for: $\langle n\rangle \sim N$. Very important, for large $\langle n\rangle$, i.e. $R \rightarrow 1$ the slope of the functions $D(x)$ increase fast towards a very large value: $R \rightarrow 1: S l=\lim _{R \rightarrow 1}|d D(x) / d x| \approx \infty$. The latter property can be demonstrated considering 


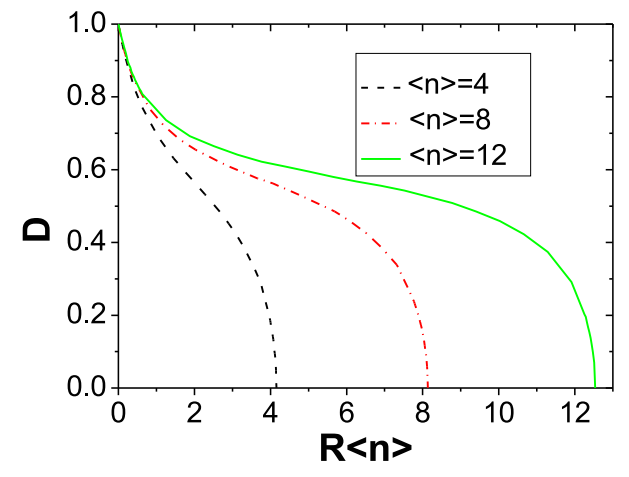

FIG. 6: (Color online) Numerical evaluation of the Bures distance $\mathrm{D}(\mathrm{x})$ between two orthogonal equatorial macro-qubits $\left|\Phi^{\phi, \phi_{\perp}}\right\rangle$ as function of the average lost particle $\left.x=R<n\right\rangle$, plotted in linear scale. Black dashed lower line refers to $g=0.8$ and hence to $\langle n\rangle \approx 4$, red dash-dotted middle line to $g=1.1$ and $\langle n\rangle \approx 8$, green straight upper line to $g=1.3$ and $\langle n\rangle \approx 12$. As the number of particles generated increases, two different decoherence regimes can be discriminated, i.e. in the low and high loss range.

the high lossy regime for $R \approx 1$, and keeping only the first order terms in $T$ of the density matrix, obtaining: $\lim _{T \rightarrow 0} \frac{\partial D\left(\hat{\rho}_{T}^{\phi}, \hat{\rho}_{T}^{\phi}\right)}{\partial T}=1+4 C^{2}+2 C^{2} \Gamma\left(1+2 \Gamma^{2}\right)^{\frac{1}{2}} \stackrel{g \rightarrow \infty}{\rightarrow} \infty$

All this means that the MQS Visibility can be large even if the average number $x$ of lost particles is close to the total number $N$, i.e. for $R \sim 1$. As seen, this behavior is opposite to the case of coherent states where the function $D(x)$ approaches zero value with zero slope: Fig.2-(e). We believe that this lucky and quite unexpected behavior is at the core of the high resilience to decoherence of our QI-OPA MQS solution. Note that this behavior was responsible for the well resolved interference pattern with visiblity: $V \approx 20 \%$ obtained in absence of O-Filter (OF) by [18]. As a trivial remark, note that in all Figures of the prseent work the function $D(x)$ necessarily drops to zero for $\langle n\rangle \simeq N$, the total number of particles associated to the macrostates.

To gain insight on this feature we report in Fig 6 the visibility between the $\left|\Phi^{ \pm}\right\rangle$states as a function of the average lost photons plotted in a linear scale. As the number of photons generated by the amplification increases, the curves allows to identify two different regimes in the decoherence process. As said, at low $R$, the rapid decay is due to the cancellation of the comb structure in the photon number distribution. On the contrary, when $R$ is progressively increased, the initial exponential decay is interrupted, a kind of plateau appears and a more resilient structure is found. This resilient portion of the spectrum is attributable to the unbalancement in the photon number distributions in the Fock space $P\left(n_{\Phi}, n_{\Phi \perp}\right)$ for the "equatorial" macrostate $\left|\Phi^{\phi}\right\rangle$ due to the polarization encoding of the seed microqubits. This kind of encod- ing is missing in the coherent-state MQS case since there the fragile MQS interference is only related to the existence of the comb structure of the Fock spectra and then quickly disappears with it, as said. In other words, two initially orthogonal polarization states maintain their distributions unbalanced in different zones of the twodimensional Fock space even in regimes of large losses. An interesting case is presented by Fig. 5. (b) that shows the rapid decay of the coherence of $D\left(\left|\Phi^{H}\right\rangle,\left|\Phi^{V}\right\rangle\right)$ under BS-scattering of the macrostates $\left|\Phi^{H}\right\rangle,\left|\Phi^{V}\right\rangle$ which do not belong to the "equatorial plane" of the Poincaré sphere. A close comparison with Fig 5 -(a) emphasizes the role of the privileged "noise reduction" Hilbert subspace with respect to decoherence of the macrostates.

Orthogonality Filter (O-Filter). The demonstration of microscopic-macroscopic entanglement by adopting the O-Filter was reported in [12]. The POVM like technique [32] implied by this device locally selects the events for which the difference between the photon numbers associated with two orthogonal polarizations $|m-n|>k$, i.e. larger than an adjustable threshold, $k$ [18]. By this method a sharper discrimination between the output states $\left|\Phi^{\phi}\right\rangle$ e $\left|\Phi^{\phi_{\perp}}\right\rangle$ can be achieved. The action of the O-Filter can be formalized through the measurement operator $\hat{P}_{O F}=\sum_{m, n}\left|m \phi, n \phi_{\perp}\right\rangle\left\langle m \phi, n \phi_{\perp}\right|$, where the sum over $m, n$ extends over the terms for which the above inequality holds. The O-Filter can be implemented experimentally either by a post-selected configuration in the measurement apparatus directly coupled to the output of the QI-OPA device or by the optical scheme represented in the Right Inset of Fig 3. There a small portion $(\simeq 10 \%)$ of the photon flux associated with the macrostate $\left|\Phi^{\phi}\right\rangle$ realized at the output of the QI-OPA is analyzed by an "idle" measurement apparatus connected to an O-Filter that activates a polarization preserving, high-voltage, fast electro-optical shutter. At last, the OFiltered macrostate $\left|\widetilde{\Phi}^{\phi}\right\rangle$, corresponding to $\left|\Phi^{\phi}\right\rangle$ emerges at the output of the apparatus [33].

We now analyze theoretically the action of the O-Filter device evaluating the Bures distance between the filtered states $\frac{\hat{P}_{O F} \hat{\rho}_{T}^{\phi} \hat{P}_{O F}^{\dagger}}{\operatorname{Tr}\left(\hat{P}_{O F} \hat{\rho}_{T}^{\phi} \hat{P}_{O F}^{\dagger}\right)}$ and $\frac{\hat{P}_{O F} \hat{\rho}_{T}^{\phi} \perp \hat{P}_{O F}^{\dagger}}{\operatorname{Tr}\left(\hat{P}_{O F} \hat{\rho}_{T}^{\phi} \hat{P}_{O F}^{\dagger}\right)}$. We then applied the numerical methods previously described to calculate the Bures distance $D(x)$ as a function of the threshold $k$. In Fig 7 the results of a numerical analysis carried out for $g=0.8$ and different values of $k$ are reported.

Note the increase of the value of $D(x)$, i.e. of the MQS Visibility, by increasing $k$ and, again: $S l=$ $\lim _{R \rightarrow 1}|d D(x) / d x| \approx \infty$. Of course, in the spirit of any POVM measurement, the high interference visibility is here achieved at the cost of a lower success probability [34]. The general, most important feature shown by all these Figures is that both the "Distinguishability" and the "Visibility" of all "equatorial" macrostates $\left|\Phi^{\phi}\right\rangle,\left|\Phi^{\phi \perp}\right\rangle$ as well as of all their "equatorial" quantum superpositions can be kept close to the maximum value in spite of the increasing effect of decoherence due to 


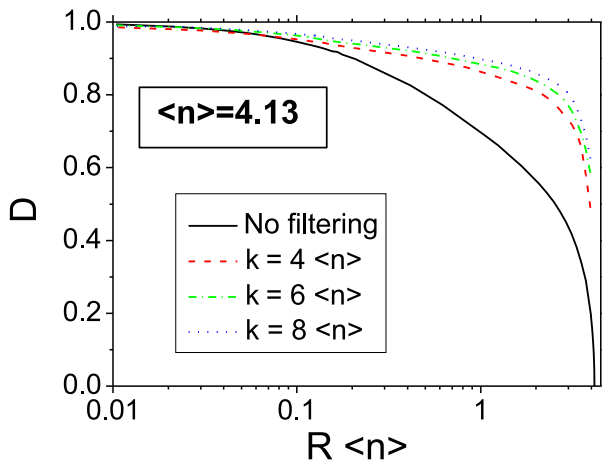

FIG. 7: (Color online) Numerical evaluation of the Bures distance between two orthogonal equatorial O-filtered macroqubits for different values of the threshold $k(g=0.8)$. Black straight lower line corresponds to the case in which the OFilter is not applied. For the remaining curves, from the bottom to the top, red dashed line corresponds to a threshold $k=\langle 4\rangle$, green dash-dotted line to $k=\langle 6\rangle$ and blue dotted line to $k=\langle 8\rangle$.

increasing values of the quantity: $R<n>$. On the basis of all these results we may then conclude that all the "equatorial" macrostates and superpositions generated by the QI-OPA may be safely defined as classically stable, einselected "pointer states" [21]. The validity of this statement has been demonstrated in the Laboratory, as shown for instance by the high visibility of the two experimental interference patterns corresponding to two different measurement orthogonal bases, and appearing in the Left Inset of Fig 3 [12].

\section{INTERPRETATION AND CONCLUSIONS}

The efficiency of the transfer of classical or quantum information in the interactive dynamics involving the paradigmatic quantum - statistical combination (System + Environment) is at the focus of the present investigation. In order to gain insight into the general picture and to support the congruence of our final conclusions we find useful to relate here the various aspects of the cloning process provided by QI-OPA with the current, most sensible MQS physical models, in particular with Zurek's one 21].

1) The "System" in our scheme is represented by the assembly of $N$ photon particles associated with any macrostate $\left|\Phi^{\phi}\right\rangle$ generated by the optical parametric method based on phase-covariant quantum cloning.

2) The flow of (classical) "noise information" directed from the "Environment" towards the System is provided in our case by the unavoidable squeezed-vacuum noise affecting the building up of the macrostate $\left|\Phi^{\phi}\right\rangle$ within the process of parametric amplification. As already stressed, the "optimality" of the phase - covariant quantum cloning adopted in our experiments implies, and literarly means, that the flow of classical noise is the minimum allowed by the principles of quantum mechanics, i.e. by the "nocloning theorem" [13, 14].

3) The flow of quantum information directed from the System towards the Environment is provided by the controlled "decoherence in action" provided by the artificial BS-scattering process adopted for our analysis as well as by the losses taking place in any realistic photo-detector. We have seen that by the use of the Orthogonality Filter, or even in the absence of it, the interference phasedistrupting effects caused by the adopted artificial decoherence can be efficiently tamed and even cancelled to a great extent for the "equatorial" macrostates and for their quantum superpositions.

4) The selected quantum cloning method, realized by the QI-OPA device, allows to define a privileged " minimum noise - minimum decoherence" Hilbert subspace of the quantum macrostates that, according to our decoherence model, exhibit simultaneously the maximum allowed Distinguishability and Visibility. According to the theory of quantum cloning a smaller size of the privileged Hilbert subspace, here the equatorial plane of the Poincarè sphere, corresponds to a higher "cloning fidelity" and then to a smaller flux of squeezed - vacuum "noise information" from the environment to the system.

5) The last paragraph of the sentence written by Einstein on the back of his greetings card to Born appears to conflict with the behavior of the system generated by our cloning apparatus. In facts, in our case quantum mechanics cannot be taken as "incompatible" with the "classical" localization of any macro-state $\left|\Phi^{\phi}\right\rangle=\hat{U}|\phi\rangle$ more than it may be for the "quantum" localization of the corresponding micro-state $|\phi\rangle$. For, in our case a unitary quantum-cloning transformation $\hat{U}$ connects, we would say "chains" albeit in a noisy manner, all physical properties belonging to the micro-world to the corresponding ones belonging to the macrosopic "classical" world. Any lack of perceiving or rationally accepting this close correspondence, for instance in connection with the realization or detection of the "Schrödinger Cat", must be only attributable to the intrinsic limitations of our perceiving senses, of our observational methods or of our measurement apparata. In other words, at least in our case, the two worlds, the Macro and the Micro are deterministically mirrored one into the other where the "mirror", albeit somewhat blurred, is provided by quantum mechanics itself. In a forthcoming paper many conceptual and theoretical aspects of the work presented here will be further analyzed in the phase-space by investigating in details the Wigner functions of the MQS generated by phase-covariant quantum cloning in presence of losses. All these results belongs to the lessons we learned recently by the experiments carried out in our Laboratory.

In summary, the present work was intended to give a somewhat firm conceptual basis to the unexpected high resilience to decoherence demonstrated in recent experiments by our QI-OPA generated Macroscopic Quantum 
Superposition. We believe that this novel MQS system may play further relevant roles in the future investigations on the foundational structure of Quantum Mechanics. We acknowledge very useful discussions and corre- spondence with Chiara Vitelli and Gunnar Bjork. Work supported by PRIN 2005 of MIUR and INNESCO 2006 of CNISM.
[1] Einstein-Born Briefwechsel 1916-1955, Nymphenburger Verlagshandlung GmbH, Munchen (1969)

[2] A. Einstein, et al., Phys. Rev. 47, 777 (1935).

[3] E. Schroedinger, Naturwissenschaften 23, $807-812$, $823-828,844-849$ (1935).

[4] M.A. Nielsen and I.L.Chuang, Quantum Information and Quantum Computation (Cambridge University Press, 2000)

[5] W. Zurek, Physics Today, October 1991, pag.36; Rev. Mod. Phys. 75, 715 (2003); Progr. Math. Phys. 48, 1 (2007)

[6] W. Dur, et al., Phys. Rev. Lett. 89, 210402 (2002) ; W. Dur, et al., Phys. Rev. Lett. 92, 180403 (2004)

[7] T. Gorin, et al., Phys. Rev. Lett. 99, 240405 (2007)

[8] W.P. Schleich, Quantum Optics in Phase Space (Wiley, New York, 2001), Chaps. 11 and 16.

[9] M. Brune, et al., Phys. Rev. A 45, 5193 (1992)

[10] J.M. Raimond,et al., Rev. Mod. Phys. 73, 565 (2001)

[11] A. Ourjoumtsev, et al., Science 382, 83 (2006); A. Ourjoumtsev, et al., Nature 448, 784 (2007)

[12] F. De Martini, et al., Phys. Rev. Lett. 100, 253601 (2008).

[13] F. Sciarrino, et al., Phys. Rev. A 72, 062313 (2005).

[14] F. Sciarrino, et al., Phys. Rev. A 76, 012330(2007).

[15] F. De Martini, Phys. Rev. Lett. 81, 2842 (1998); Phys. Lett. A 250, 15 (1998).

[16] F. De Martini et al., Prog. Quant. Elect. 29, 165 (2005).
[17] F. De Martini, et al., Phys. Rev. Lett. 95, 240401 (2005).

[18] E. Nagali, et al., Phys. Rev. A 76, 042126 (2007).

[19] M. Ricci, et al., Phys. Rev. Lett. 95, 090504 (2005)

[20] F. De Martini, et al., arXiv:0804.0341.

[21] W.H. Zurek, Phys. Rev. Lett. 90, 120404 (2003).

[22] R. Jozsa, J. Mod. Opt. 41, 2315 (1994)

[23] D. Bures, Trans. Am. Math. Soc. 135, 199 (1969)

[24] M. Hubner, Phys. Lett. A 163, 239 (1992); Phys. Lett. A 179, $226(1993)$

[25] R. Loudon, The Quantum Theory of Light.

[26] U. Leonhardt, Phys. Rev. A 48, 3265 (1994)

[27] W. Schleich, et al., Phys. Rev. A 44, 2172 (1991)

[28] The closed-form calculations leading to Eq.3 as well as similar expression for $D(x)$ will be reported in a forthcoming paper.

[29] G. Bjork, private communication

[30] J. Jacobson, et al., Phys. Rev. Lett. 74, 4835 (1995); K.T. Kapale, et al., Phys. Rev. Lett. 99, 053602 (2007); F. Sciarrino, et al. Phys. Rev. A 77,012324 (2008).

[31] L.J. Slater, Generalized Hypergeometric Functions, Cambridge University Press, Cambridge (1966)

[32] A. Peres, Quantum Theory: Methods and Concepts (Kluwer Academic Publishers, Dordrecht,1995).

[33] N. Spagnolo, et al., Optics Express 16, 17609 (2008)

[34] B. Huttner, et al., Phys. Rev. A 54, 3783 (1996) 